\title{
Region-based Image Segmentation Using Shape-Varying Agents Xu Qi
}

Dept. of Computer Science and Technology School of Information Science and Engineering Shanghai Maritime University, Shanghai, P.R. China, 201306

qixu@shmtu.edu.cn

Keywords: Image segmentation, Alife, Shape-Varying Agent

\begin{abstract}
Image segmentation is an important problem in image processing. Existing methods have not solved this problem in some aspects. Alife is a new natural computing method with much good performance. We induce it into image segmentation and propose a novel Alife model Shape-Varying Agent (SVA). The new method combines the advantages of Alife approaches and region growing approaches, achieving good performance on image segmentation.
\end{abstract}

\section{Introduction}

Image segmentation plays an important role in image processing tasks. In current computer vision, image segmentation is the basement for many other processing. It is helpful for coding, classification, recognition and so on.

Research on gray image segmentation has gone through several decades, numerous methods have been proposed. They are broadly divided into four categories: threshold methods, boundary based methods, region based methods and hybrid methods. Threshold techniques use histograms to achieve threshold segmentation. It supposes that all pixels whose values are within a certain range belong to the same class. Adaptive threshold and optimal threshold [1] generally need a few calculations and can be completed very easily. Choosing the right threshold is the key to these methods and neglecting all the spatial information of images is the fatal shortage [8]. Boundary based methods depend on edge detection operators. Such operators assume the pixel values rapid change at the boundary between two regions. The well known gradient operators are Sobel filter [2] and Roberts filter [3]. But the results of edge detection are often discontinuous which must be modified to be closed curves to represent the boundaries. The process is a difficult task and these methods do not cope well with noise [8]. Region based methods depend on region homogeneity. The basic idea is to segment the image into the biggest homogeneous regions as large as possible. Typical algorithms are split-and-merge [4], Morphological watershed [5] and Texture analysis [7]. It take account of the spatial information and relatively robust for noises. However, it is difficult to detect edges and choose an appropriate homogeneity criterion. Expensive computation is another problem for region based method. Hybrid methods generally are mixture of some methods. Recently, Alife has been applied to image segmentation [6] and achieves some cheering results.

In this paper, we present a novel method termed region based segmentation by shape-varying agents (RSVA). Traditional agent always is a pixel without structure and individual behaviors. Generally, they combine Alife and region growing methods to get segmentation results. The biggest improvement of RSVA is that each agent has its own shape and never be restricted to a pixel size. Agents in different region may have different shape and size. Therefore, shape-varying agents become "Segmentation Primitives" like "Texture Primitives" to some extent. We can get more spatial information and efficient results by shape-varying agent (SVA). 


\section{Model of Shape-Varying Agents}

There are many methods utilizing agents for segmentation [8,9]. Most of them take a pixel as an agent for region growing. Apparently, they cannot deal with nature image very well (e.g. grass image, leaves image). For example, in a nature image, we just want to know where the grass is, where the tree is or where is the sun. Most of us will not to concern every pieces of grass or every piece of leaves. In these situations, one-pixel agents always are over segment. They cannot solve it very well.

In RSVA, shape-varying agents (SVA) which can include more information (like texture primitives) are distributed in every different region of the image; they can grow into any shape in order to typically delegate the features of this region. These SVAs make the image to be texture homogenous and similar ones with identical structure features will be unified to construct the homogenous image region as large as possible. Therefore, it can avoid over segmentation very easily. Individual structure and behavior render shape-vary agent more intelligent than any other agents. On the other hand, we think if an agent finds its proper living environments at some direction, it will move on at this direction to expend its living fields. Whereas, differing from the stochastic strategy of most Alife methods, SVA adopts inertial strategy for agents' movements.

The image $\mathrm{R}$ is considered as the living environment of SVAs, different subregion Error! Reference source not found. has different living conditions. A SVA suits for the subregion Error! Reference source not found. where it born. $R=\left\{R_{1}, R_{2}, R_{3} \cdots R_{i} \cdots R_{n}\right\}, R_{i} \cap R_{j}=\phi$, for every $\mathrm{i}$

and $\mathrm{j}, i \neq j$. SVA is greedy. It is inspired to expand its field as large as possible. The process can be divided into two parts: occupying and unifying. Occupying phase is an outer instruction, including growth, outspread and split. Unifying phase mainly has four instructed regulations and regarded as an intern instruction. More details will be discussed in the following sections.

\subsection{Occupying Process}

This phase mainly solve the segmentation among agents. We take it as outer instruction because it works among different classes of agents. In this phase, SVAs move as subsequent three rules:

(1) Growth.

The growth rule is the most important rule in RSVA. Now that we call SVA "segmentation primitive", it must present the features of segments. Similar to "Texture Primitives", SVA is hypostatic, shaped and local. To design the SVA, the Error! Reference source not found.-radius neighborhood is firstly defined,

Definition 2.1 The r-radius neighborhood $N_{P(s, t)}^{r}$ of a SVA is

$$
N_{P(s, t)}^{r}=\left\{Q(u, v) \mid \max (|u-s|,|v-t|) \leq r, Q(u, v)_{-} \text {size }=P(s, t)_{-} \text {size }\right\}
$$

where Error! Reference source not found. is the position of SVA, $r$ is the distance between two SVAs (e.g. $r=1$ means that the neighbors of agent $\mathrm{P}(\mathrm{s}, \mathrm{t})$ is its 8 neighbors which has identical shape and identical size).

Definition 2.2 Each SVA is designed to represent the features of its neighborhoods. The growth rule is depicted as

$$
\begin{gathered}
P^{n+1}(s, t)_{-} \text {size }=P^{n}(s, t)_{-} \text {size }+F\left(P^{n}(s, t), Q^{n}(u, v)\right) \\
F(P(s, t), Q(u, v))=\text { Error! Reference source not found. }
\end{gathered}
$$


If $\mathrm{P}(\mathrm{s}, \mathrm{t})$ is similar with most neighbors, then we think it can delegate local features:

$R_{1}(P(s, t), Q(u, v)) \geq \varepsilon, R_{2}(P(s, t), Q(u, v)) \leq \eta$

Where $Q(u, v) \in N_{p(s, t)}, \varepsilon>0$ and $\eta>0 . R_{1}()$ and $R_{2}()$ are regularity functions. They are defined as:

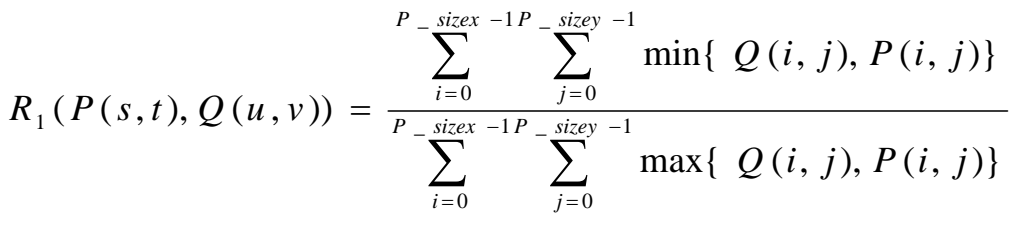

$$
\begin{aligned}
& R_{2}(P(s, t), Q(u, v))=\left|\delta_{p}-\delta_{Q}\right| \\
& \delta^{2}=\frac{1}{P(s, t)_{-} \text {size }} \sum_{i=0}^{P-\text { sizex }-1 P} \sum_{j=0}^{\text {sizey }-1}(P(i, j)-\overline{P(s, t)})^{2} \\
& \overline{P(s, t)}=\frac{1}{P(s, t)_{-} \text {size }} \sum_{i=0}^{P-\text { sizex }-1 P} \sum_{j=0}^{\text {sizey }-1} P(i, j)
\end{aligned}
$$

where $P(s, t)$ and $Q(u, v)$ are patterns of SVA. $P(i, j)$ is the energy value of $I(s+i, t+j), Q(u, v)$ is the energy value of $I(u+i, v+j)$ and $I(i, j)$ is the intensity value of the pixel at the position $(i, j)$.

We use a two-dimensional regularity measurement to describe the SVAs and their placement rules. Agents in different regions may be evolved different shape and has local features.

(2) Outspread.

After SVAs have matured into "segmentation primitives", they begin to expand their living field. Outspread rule adopts inertial strategy. If a SVA get a new field in some direction at last time, then it go on. Outspread rule simulates snail's crawl, if its first expanding encounter un-homogeneity region, then it shrink a little, when it can't shrink any more, it change the direction. The feeler of a SVA simulates the antenna of snails in expanding process. If the feeler feels the region suitable, then the SVA occupy it and leave hormones (or footmarks) which make other SVAs get away. If the feeler find the region unsuitable, then the feeler shrink a part, and make itself has some overlaps with its head. The process is illustrated in Fig. 1.

$$
P(s, t)_{\text {feeler }}=\left\{Q(u, v) \mid Q(u, v) \in N_{P(s, t)}^{r}, r=P(s, t)_{-} \text {size }-d\right\}
$$

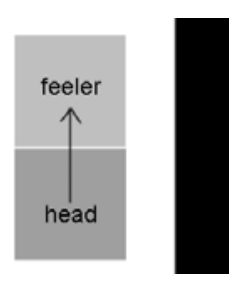

(a)Homogeneous head and feeler
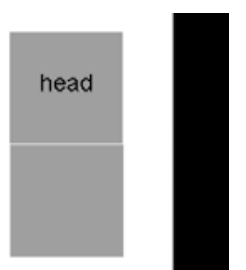
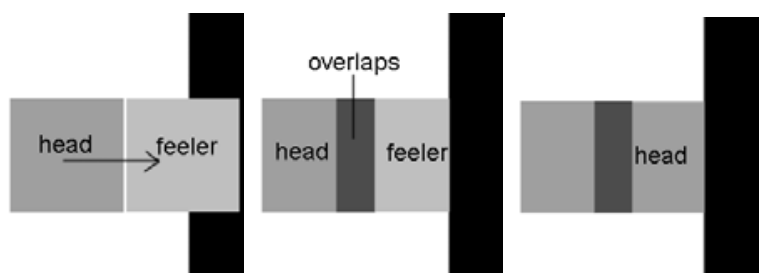

(a)Un-homogeneous head and feeler

Figure 1. Refractivity of Outspread Rule. White background and black background denote two inhomogeneous regions. The squares denote SVA and its feeler.

(3) Split.

Competition is cruel in nature, and living environment is the leading cause of mutation. Insects will not waste any chance to expand its fields. Thus, split often occurs when it find a 
unsuitable field (because it expect its children can fit the region). In our model, the mutation is induced by external factors and that are different properties of the image regions. When SVA encounter un-homogeneity region, it split a seed to that region. The new seed will mature as above growth rule to become a new SVA. The new SVA has different characteristic from its mother which fit the new region and will expand its fields in this new region.

\subsection{Unifying Process}

After the occupying process, the image is segment by thousands of SVAs. In order to make the segmentation result psychically reasonable, we need to classify these SVAs. Therefore, unifying process is essential and very important. Obviously, unifying process is based on the occupying process. At the end of the procedure, the strongest segmentation primitive will unify its group which means other segmentation primitives with identical living environment will be labeled as a same class. The power of the agent is determined by its energy.

The unifying process is a dynamic procedure, and all primitive agents randomly crawl in the whole landscape. In this session, SVAs transport as following four rules:

(1) If it finds a suitable land occupied by a weaker agent, and then invade the land, mark the weaker one by its label and its energy will be increased.

(2) If it finds a suitable land occupied by a stronger agent, then go through the land with the reduction of the energy. It cannot change the label of the stronger one.

(3) If the exploring region is unfit for the agent, then it shrinks the size of the feeler and test it again. The agent changes its direction unless the feeler cannot shrink anymore. Fig. 2 illustrates that the invading activity also is flexible as expanding activity.

(4) If the agent crawls in its land, it keeps its energy and does it again.

Figure 2. The shrink procedure of invading activity.
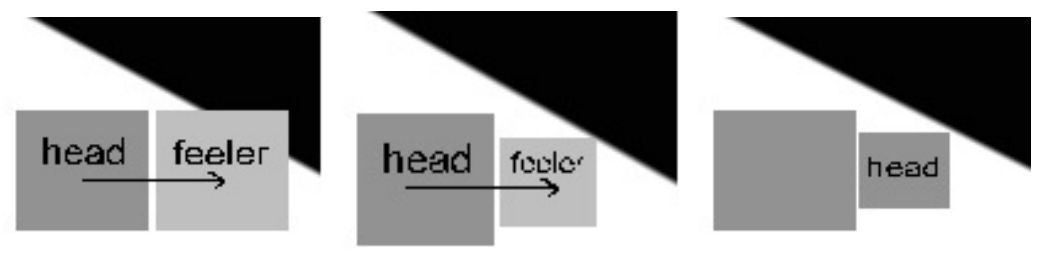

The homogeneity of unifying process is defined by $\Delta m$ and $\Delta \delta,\left|m_{i}-m_{j}\right|<\alpha,\left|\delta_{i}-\delta_{j}\right|<\beta$, where Error! Reference source not found. and Error!

Reference source not found. are threshold parameters to control the scale of unifying.

\section{Experiments and Analysis}

We applied the model of SVA in image segmentation, and the steps of experiments are guided as follows:

(1) Initial. The whole image is considered as the living environment of SVAs. Enough seeds of SVAs are arbitrarily distributed around the image, and each seed get instinctive feature because of the gray intensity and spatial structure of its born region. We set the ith seed has initial energy $E_{i}=20$.

(2) Occupying Process. Every seed firstly becomes segmentation primitive as growth rule, and then it begin to outspread and split. If the seed successfully occupied a new region, it get two bonus 
energy. The process stops when all pixels of this image have been occupied.

(3) Unifying Process. Unifying activity occurs after occupying. Strong SVA will get more land than weak one in its group, and get one bonus energy unit when it gets a new region. However, if the weak SVA invades the land of strong one, it will lose two energy units and die with zero energy. After this process, we will get the biggest homogenous region and complete the image segmentation.

Fig. 3 shows the experiment result. RSVA is a supervised method, agent seeds (time $=1$ ) are not a pixel but a kind of texture primitive which is the breakthrough of this method. Fig. 3(e) is the end of occupying process (time=10). After a while of unifying, some of the land are merged by strong SVAs (Fig. 3(h) shows the unifying result at time=500). The agent of RSVA has its own structure features and make the segmentation can be carried out at semantic level. In one hand, the agent can grow into a series primitive with different size according to its structure. This property will prominently enhance the performance of texture image segmentation. In the other hand, the segmentation scale can be controlled by the size of SVA. This property is helpful for over segmentation and insufficient segmentation.
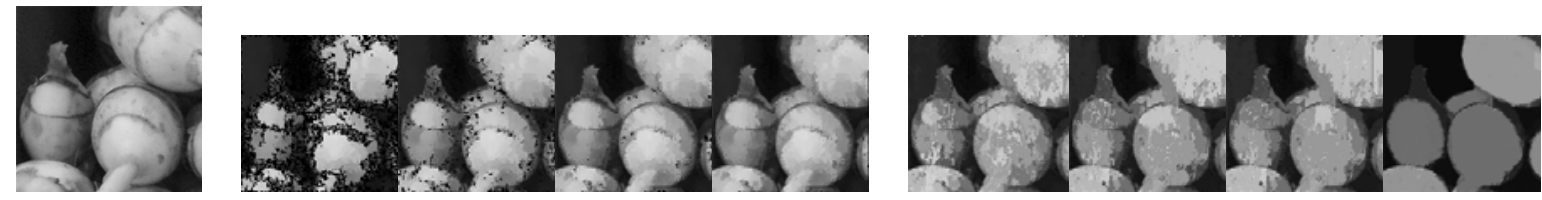

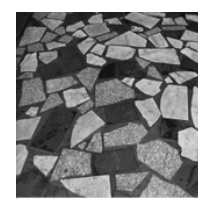

(a)

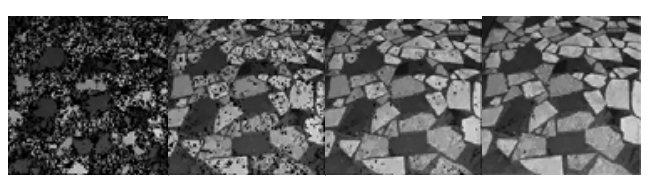

(b) (c) (d)

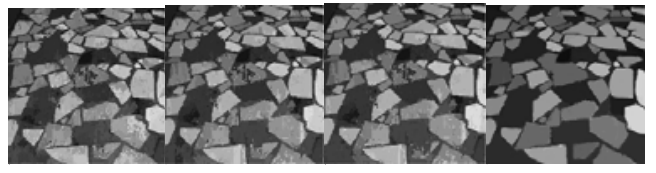

(f) (g) (i)

Figure 3. (a) The original image; (b)-(e)The procedure of occupying: $R_{1}=0.85, \quad R_{2}=2$, $E=20$ (b)time=1; (c)time=5; (d)time=10; (e)The result of occupying; (f)-(i)The procedure of unifying: $\Delta m=10 ; \Delta \delta=8$; (f)time $=10$; (g)time=50; (h)time=500; (i)Segmentation results 。

Fig. 4 illustrates the compared results with Split-and-Merge method and GMRF method. RSVA is obviously better than others. The results of Split-and-merge method only obtained rough segmentation results. The boundaries are not natural. GMRF method cannot keep the image details and most boundary information. In opposite, the agents of RSVA can suitable for different regions with different size which make the segmentation results reasonable with smooth boundaries.

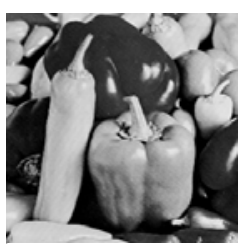

(a)

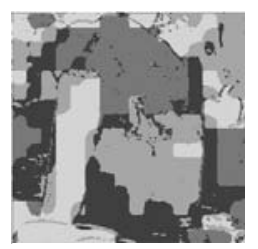

(b)

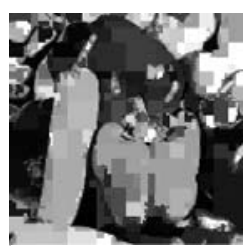

(c)

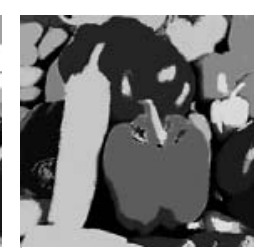

(d)

Figure 4. Comparison results for image segmentation。(a)The original images; (b) The results of GMRF method; (c) The results of Split-and-Merge method; (d)The results of RSVA method。

\section{Conclusion}

We propose a novel method--SVA solve image segmentation and get some good performances. Image segmentation has no fix benchmark for different person which means maybe we should make the segmentation approach is supervised at semantic level. RSVA is designed on this purpose and it 
overcome the shortage of existing artificial life methods that agents always is a pixel and have no individual behaviors. Artificial life is not confined to any concrete forms, how to utilize multi-dimension life to multiscale image segmentation is our following works.

\section{References}

[1] C.A. Glasbey, An Analysis of Histogram-Based Thresholding algorithms, CVGIP-Graphical Models and Image Processing, 55:532-537, 1993.

[2] J.F. Canny. A Computational Approach to Edge Detection. IEEE Transactions on Pattern Analysis and Machine Intelligence, 8(6): 679-698, 1986.

[3] L.G. Roberts, Machine Perception of Three-Dimensional Solids, In J T Tippett, editor, Optical and Electro-Optical Information Processing, PP 159-197, MIT Press, Cambridge, MA, 1965.

[4] S.L. Horowitz and T Pavlidis, Picture Segmentation by a Directed Split-and-Merge procedure, In Proceedings of the 2nd International Joint Conference on Pattern Recognition, PP 424-433, Copenhagen, Denmark, 1974.

[5] L. Vincent and P. Soille, Watersheds in Digital Spaces: An Efficient Algorithm Based on Immersion Simulations. IEEE Transaction on Pattern Analysis and Machine Intelligence, 13(6): 583-598, 1991.

[6] Hao He and Yan Qiu Chen, Artificial Life for Image Segmentation, International Journal of Pattern Recognition and Artificial Intelligence, VOL.15, NO 6(2001), 989-1003.

[7] Cecilia Di Ruberto, Giuseppe Rodriguez and Sergio Vitulano, Image Segmentation by Texture Analysis.

[8] Hideki Kagawa, Makoto Kinouchi, and Masafumi Hagiwara, ImageSegmentation by Artificial Life Approach using Autonomous Agents,IEEE,1999.

[9] Cor J.Veenman, Marcel J.T.Reinders and Eric Backer A Cellular, Coevolutionary Algorithm for Image Segmentation, IEEE Transactions on Image processing, VOL.12, NO.3, MARCH 2003. 